\section{Décès imputables} à une erreur médicale
en milieu hospitalier

Prise de position de I'OFAS

O. Piller

L'article du Professeur Reto Tscholl [1] critique l'extrapolation effectuée par l'OFAS pour estimer le nombre des décès dus à une erreur médicale dans les hôpitaux suisses. Ces chiffres ont été largement diffusés, je le regrette, sans nuance ni différenciation. D'où une certaine mauvaise humeur, bien compréhensible, au sein du corps médical. J'aimerais vous faire connaître ma position, afin que, au lieu de tourner en une guerre de chiffres, cette affaire serve de tremplin à une réflexion commune en vue de résoudre un problème que le corps médical connaît bien.

\section{Tout décès est de trop}

L'OFAS n'a jamais affirmé qu'il y avait en Suisse "chaque année 3000 décès qui seraient dus à des erreurs médicales dans les hôpitaux". Notre communiqué de presse du 22 septembre 2000 fait référence au

Correspondance

Otto Piller

Directeur

Office fédéral des assurances sociales (OFAS)

Effingerstrasse 20

CH-3003 Berne

E-mail: otto.piller@bsv.admin.ch rapport, publié début 2000, par l'Institut de médecine de Washington (USA) qui dresse un tableau de la fréquence des erreurs médicales. Se fondant sur différentes études, les experts américains concluent que souvent un décès de patient survient consécutivement à une erreur médicale. Partant de l'idée que la fréquence des erreurs médicales n'est pas sensiblement différente en Suisse de ce qu'elle est aux EtatsUnis, en Australie et en Angleterre, une extrapolation permet d'avancer que chaque année 2000 à 3000 décès ont pour origine une erreur médicale. Nous n'avons jamais affirmé que la situation était identique en Suisse et aux Etats-Unis et nous avons toujours pris la précaution d'indiquer que les chiffres publiés correspondaient à une extrapolation. Faute de statistique, nous voulions, par cette estimation faire ressortir le fait que les erreurs médicales nous concernaient également et que nous avions la possibilité, le devoir et la volonté d'améliorer la situation. Qu'il y ait 30, 300 ou 3000 décès, là n'est pas vraiment la question, chaque décès est un décès de trop. Les erreurs médicales méritent d'être prises au sérieux, leur nombre peut et doit diminuer.

\section{Regrouper les forces}

Le nombre des réactions montre que l'erreur médicale et la sécurité du patient préoccupent le corps médical, ce dont je me réjouis. Depuis longtemps, les médecins prennent des mesures pour détecter et analyser les erreurs, ce qui constitue la base indispensable à toute réduction du taux d'erreurs. Il faut maintenant poursuivre ces efforts et regrouper nos forces pour nous assurer une efficacité maximale. Je me réjouis donc de travailler avec des médecins motivés et je souhaite que nous ne gaspillions pas nos énergies en de vaines querelles de chiffres.

\section{Référence}

1 Tscholl R. Hungersnot in der Schweiz nach Berechnungsmethoden des BSV. Schweiz. Ärztezeitung 2001;81:264. 